\title{
THE POLYGENIC CONTROL OF OUTBREEDING IN BORAGO OFFICINALIS
}

\author{
LESLIE K. CROWE \\ The Department of Botany, University of Reading, England
}

Received 29.viii.70

\section{INTRODUGTION}

Genetical analysis of incompatibility systems in plants has been concentrated on those species that advertise their properties by complete selfincompatibility. These obligate outbreeding systems are common and widespread, but they represent an extreme condition. There are numerous other species that are predominantly but not exclusively outbreeding. Investigation of the genetics of mating behaviour in these facultative systems has been neglected. Usually in such cases individuals are self-compatible, but this does not rule out the possibility that weak self-incompatibility exists. Estimations of compatibility are normally based on differential pollen tube growth and seed set. These criteria are useful only so long as the difference between compatible and incompatible matings is large. The method is not reliable where small differences such as might occur in a weak incompatibility system are critical.

The limited studies on facultative as opposed to obligate outbreeding systems have revealed how an incompatibility system can die by stages (Lewis and Crowe, 1956; Pandey, 1968). More extensive studies may throw some light on how incompatibility is born. It is unlikely that any outbreeding system will completely eliminate self-fertilisation at its inception. Refinement of an initially crude device will depend on appropriate adjustments in the genotype gradually increasing the efficiency of the barrier to selfing. Thus birth, like death, may be the result of a complex series of evolutionary stages, but culminating in obligate outbreeding. Before this end-point is reached, individuals may be self-compatible to a greater or lesser degree and would be eligible for inclusion in the category of facultative outbreeders.

Despite the diversity of outbreeding systems in the Angiosperms, it is unusual to find more than one type of system within a family. It is this uniformity within families and even in related families that leads to the conclusion that the systems are as old, if not older, than the families themselves (Crowe, 1964). The Boraginaceae is one of the exceptions to the general rule. It is a versatile family. Three different kinds of outbreeding system are known to exist amongst its members. Echium is gynodioecious, Pulmonaria is heterostyled whilst homomorphic self-incompatibility occurs in Symphytum, Myosotis and Borago. In such a mixed family the various systems may be comparatively recent in origin. New systems may still be evolving. In Borago officinalis itself the breeding system is not completely stabilised. East (1940) reported it to be self-incompatible, but some populations are only facultative outbreeders. The purpose of this paper is to present an account of the behaviour of these indeterminate populations. 


\section{Materials AND METHODS}

Seed was obtained from three sources:

1. The Botanic Garden, Oxford.

2. Maidensgrove, Oxfordshire.

3. Bleré, Normandy, France.

Plants were raised and pollinated in an insect-proof greenhouse except when stated otherwise in the text. Buds were emasculated 2 days before pollination. The degree of compatibility was assessed on the results of at least five replicate pollinations. Results are expressed as percentages of seed set since each flower produces a maximum of four nutlets.

Observations on pollen tube growth were carried out 24 hours after pollination. The styles were cut in half, stained in cotton blue and squashed.

When two kinds of pollen were tested on the same style the stigma was slit with a razor blade. The two halves were separated by a thin strip of polythene and pollinated individually.

\section{Self-FERTilisation}

Fifty-three plants of $B$. officinalis were raised from seed. Only three of these were strictly self-incompatible. The rest showed various degrees of self-compatibility ranging from 5 per cent. seed set after self-pollination at one extreme to 95 per cent. seed set at the other (fig. 1, A). The distribution of classes was continuous, plants with intermediate levels of self-compatibility being the most frequent. Since plants from different sources behaved alike they were treated as a single group.

The progeny of two of these plants were selected for further testing. The first parent gave 5 per cent. seed set after selfing. Its progeny were either weakly self-compatible or self-incompatible (fig. 1, B). Not only was the number of classes reduced but there was deviation from a normal distribution due to an excess of the self-incompatible class. A second inbred generation was raised from this family again selecting a weakly self-compatible plant as parent. The results of self-pollinating its progeny were in accord with the pattern established by the $\mathrm{F}_{1}$ but were more extreme (fig: $1, \mathrm{C}$ ). The proportion of self-incompatible plants was even larger and the number of classes showed a further reduction.

The second parent selected from group A gave 50 per cent. seed set after self-pollination. Its progeny showed continuous variation in self-compatibility over a wide range (fig. 1, D). Three members of the family representing various levels of self-compatibility were chosen as parents of the next generation. As the level of self-compatibility of the parents increased, so too did the spread of variation amongst the offspring. As the variability in the family increased, the proportion of self-incompatible individuals declined (fig. 1, E, G and $\mathrm{H}$ ).

Three conclusions may be drawn from the results of inbreeding. Firstly, the progeny of weakly self-compatible plants are less variable than the progeny of more highly self-compatible parents and include a higher proportion of self-incompatible individuals. Therefore there is a correlation between self-incompatibility and homozygosity on the one hand and selfcompatibility and heterozygosity on the other. 
Secondly, self-compatibility is a quantitative character. The wide and continuous variation between and within families cannot be attributed to a

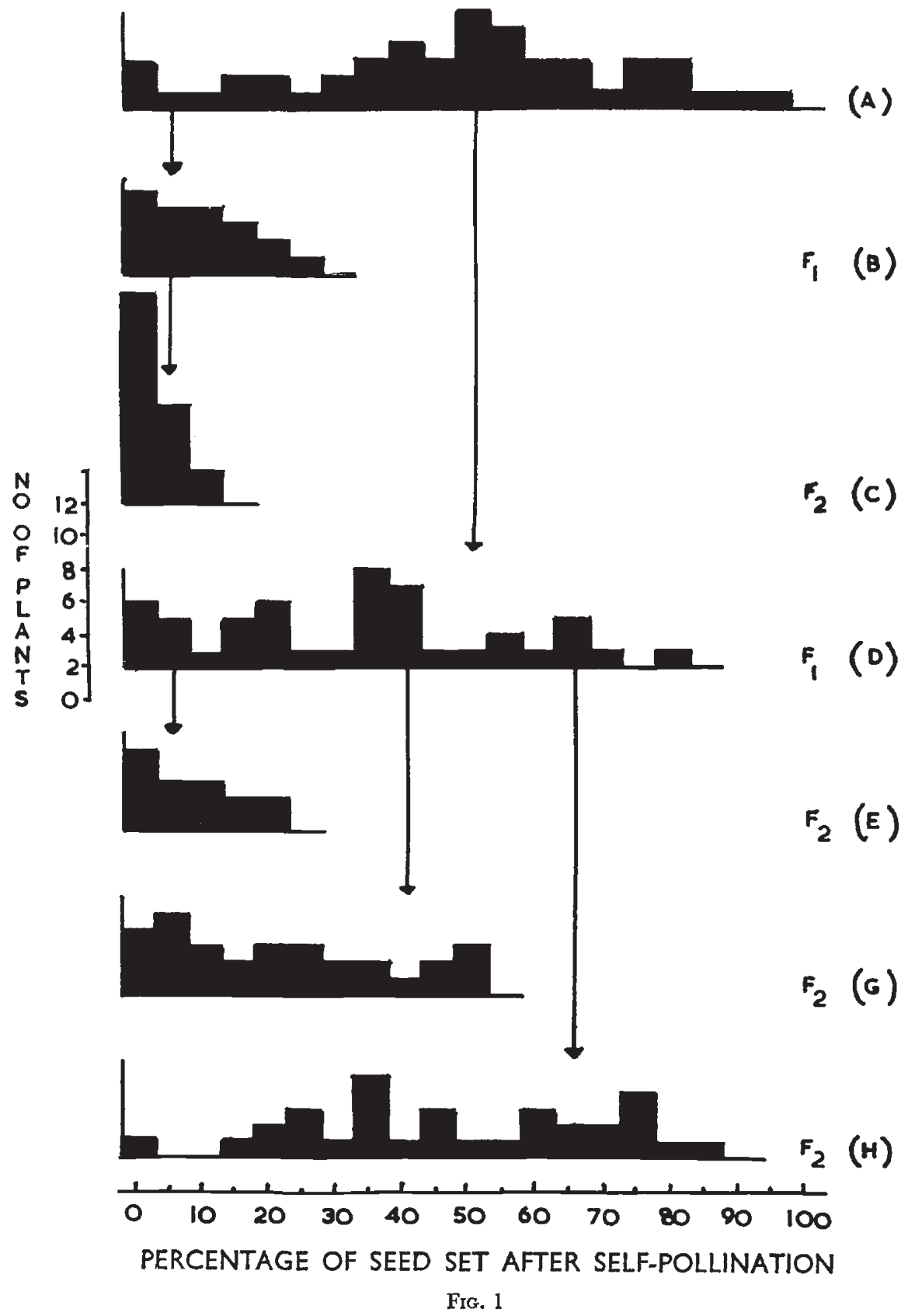

small number of genes. Thus self-compatibility is controlled by polygenes in the sense that Mather used the term (Mather, 1941).

Finally, with polygenes involved in the determination of compatibility, 
it is possible that there is a threshold effect for the incompatibility reaction. Homozygosity at more than a critical number of loci may result in complete self-incompatibility. Many different genotypes would have the necessary level of homozygosity and would fall into this self-incompatible category. This would account for the markedly skew distribution of classes in some inbred families created by the excess of self-incompatible plants. Heterozygosity in the self-compatible plants will vary in degree. The more heterozygous they are the more self-compatible they become.

\section{Cross-fertilisation}

Two of the completely self-incompatible plants from group A (fig. 1) were crossed. The plants came from different populations. A representative

\begin{tabular}{rrrrrrrrrrrrr} 
․ & \multicolumn{1}{c}{1} & 2 & 3 & 4 & 5 & 6 & 7 & 8 & 9 & 10 & 11 & 12 \\
1 & 15 & 20 & 15 & 15 & 20 & 40 & 10 & 15 & 55 & 30 & 15 & 55 \\
2 & 30 & 15 & 75 & 15 & 85 & 70 & 45 & 25 & 20 & 15 & 15 & 65 \\
3 & 40 & 30 & 15 & 5 & 50 & 15 & 65 & 60 & 25 & 80 & 50 & 50 \\
4 & 35 & 15 & 70 & 20 & 45 & 50 & 5 & 25 & 75 & 25 & 25 & 15 \\
5 & 75 & 10 & 10 & 50 & 25 & 15 & 15 & 15 & 25 & 60 & 70 & 30 \\
6 & 5 & 30 & 30 & 55 & 40 & 25 & 10 & 25 & 75 & 15 & 25 & 80 \\
7 & 20 & 35 & 15 & 10 & 10 & 80 & 30 & 20 & 50 & 25 & 15 & 10 \\
8 & 55 & 60 & 45 & 60 & 25 & 50 & 25 & 40 & 20 & 40 & 30 & 60 \\
9 & 25 & 65 & 95 & 85 & 10 & 30 & 45 & 30 & 35 & 70 & 35 & 15 \\
10 & 10 & 70 & 50 & 15 & 65 & 40 & 75 & 15 & 55 & 55 & 35 & 80 \\
11 & 50 & 40 & 45 & 50 & 50 & 75 & 70 & 55 & 30 & 10 & 60 & 15 \\
12 & 25 & 20 & 10 & 45 & 45 & 35 & 20 & 10 & 25 & 60 & 85 & 15
\end{tabular}

FIG. 2. - The compatibility of self and cross-pollinations of sibs descended from a cross between two self-incompatible plants. The compatibility is expressed as the percentage of seed set.

sample of twelve plants in the progeny were selfed and intercrossed in all possible combinations. None of the pollinations was incompatible (fig. 2). The levels of compatibility in both self and cross-pollinations were highly variable and it proved impossible to find any two individuals with the same pattern of mating.

Progeny of a cross between two sibs from this $F_{1}$ family were retained. Ten of the progeny were selfed and intercrossed. The close relationship of the parents is reflected in the self-incompatibility or weak self-compatibility of their offspring. Examples of cross incompatibility were also found (fig. 3). Some reciprocal crosses gave strikingly different results. For example, $5 \times 6$ produced a seed set of 50 per cent. while $6 \times 5$ was incompatible. Still none of the plants were alike in their mating behaviour.

Ten plants from family G (fig. 1) were also self- and cross-pollinated. These individuals were the product of two generations of self-fertilisation. 
Seven were completely self-incompatible and a high proportion of the crosses were incompatible. The residual heterozygosity was still sufficient to produce 10 unique mating types (fig. 4).

\begin{tabular}{|c|c|c|c|c|c|c|c|c|c|c|}
\hline & 1 & 2 & 3 & 4 & 5 & 6 & 7 & 0 & 9 & 1 \\
\hline 1 & 5 & 0 & 5 & 25 & 25 & 25 & 25 & 15 & 50 & 3 \\
\hline ? & 0 & 0 & 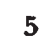 & 35 & 15 & 15 & 15 & 35 & 50 & 1 \\
\hline 3 & 15 & 0 & 5 & 50 & 10 & 0 & 60 & 25 & 20 & 2 \\
\hline 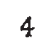 & 4 & 0 & 5 & 15 & 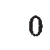 & 0 & 25 & 30 & 50 & \\
\hline 5 & 60 & 35 & 10 & 0 & 5 & 50 & 25 & 50 & 25 & 15 \\
\hline 6 & 45 & 50 & 25 & 20 & 0 & 15 & 15 & 50 & 40 & 4 \\
\hline 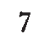 & 15 & 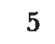 & 25 & 25 & 0 & 0 & 20 & 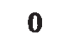 & 10 & \\
\hline 8 & 30 & 20 & 20 & 15 & 20 & 0 & 5 & 10 & 5 & 6 \\
\hline 0 & 40 & 40 & 50 & 0 & 15 & 25 & 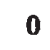 & 0 & 0 & \\
\hline & 25 & 40 & 25 & 55 & J & 15 & 5 & 30 & 15 & \\
\hline
\end{tabular}

Fig. 3. - The compatibility of self- and cross-pollinations of the progeny of a cross between two self-compatible sibs. Compatibility is expressed as the percentage of seed set. Heavy type indicates reciprocal differences in compatibility.

The first fact emerging from the results of cross-pollination is that there is self-compatibility and segregation amongst the offspring of two completely self-incompatible parents. Therefore self-incompatible individuals

\begin{tabular}{|c|c|c|c|c|c|c|c|c|c|c|}
\hline \\
\hline & 1 & 2 & 3 & 4 & 5 & 6 & 7 & 8 & 9 & 1 \\
\hline 1 & 0 & 0 & 0 & 0 & 0 & 0 & 0 & 15 & 0 & \\
\hline 2 & 0 & 0 & 0 & 0 & 5 & 0 & 20 & 0 & 0 & \\
\hline 3 & 0 & 0 & 0 & 0 & 0 & 0 & 0 & 0 & 10 & \\
\hline 4 & 0 & 15 & 5 & 0 & 0 & 0 & 0 & 0 & 0 & \\
\hline 5 & 0 & 5 & 0 & 5 & 0 & 0 & 5 & 0 & 5 & \\
\hline 6 & 0 & 0 & 0 & 0 & 0 & 0 & 0 & 0 & 0 & \\
\hline 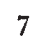 & 15 & 0 & 0 & 0 & 5 & 0 & 0 & 0 & 0 & 20 \\
\hline 8 & 0 & 0 & 15 & 5 & 0 & 10 & 0 & 5 & 0 & \\
\hline 9 & 0 & 0 & 0 & 0 & 0 & 20 & 0 & 0 & 5 & \\
\hline & 0 & 5 & 0 & 0 & 15 & . & 0 & 15 & 0 & \\
\hline
\end{tabular}

Fig. 4.-The compatibility of self- and cross-pollinations between sibs resulting from two generations of self-fertilisation. Compatibility is expressed as the percentage of seed set.

are genetically different and not necessarily completely homozygous. Thus there is further evidence in support of the threshold concept of selfincompatibility. 
Secondly, the failure of the majority of compatible pollinations to produce the full complement of four seeds suggested that the incompatibility reaction may be expressed after fertilisation resulting in the abortion of potential seeds. Observations of pollen tube growth lent support to this theory. Twenty-four hours after pollination, pollen tubes were found at the base of the style in both compatible and incompatible crosses. The results of double pollinations were conclusive. One-half of the stigma of self-incompatible individuals was self-pollinated. Compatible pollen was applied to the other half after an interval of 24 hours. Ten self-incompatible

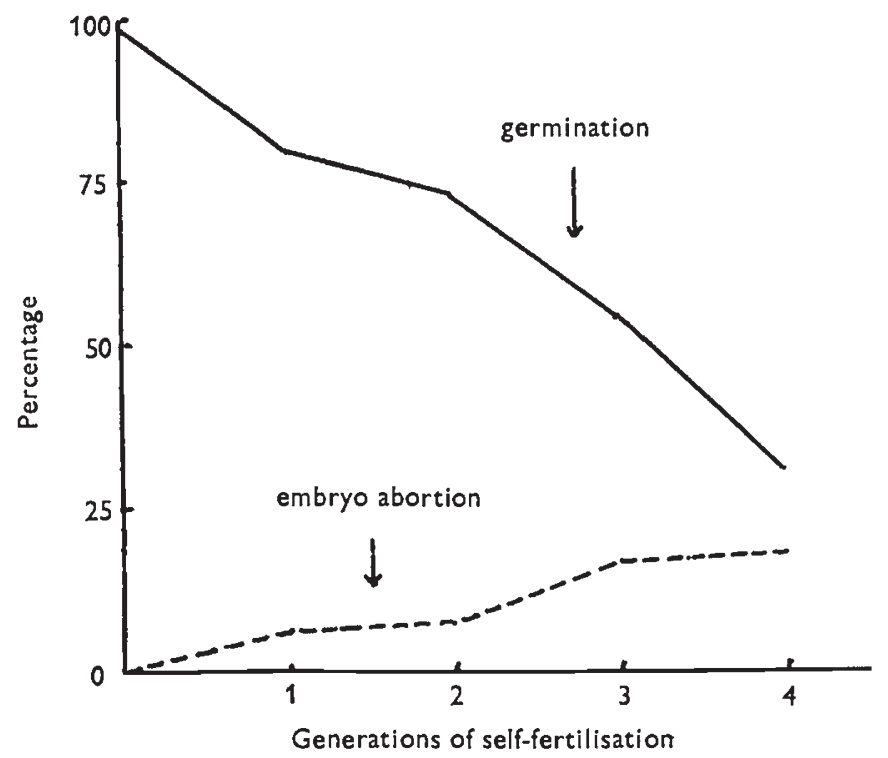

Frg. 5

plants were involved in the tests. Sixteen flowers of each plant were used. Only seven seeds were obtained from eighty doubly pollinated flowers as opposed to 149 from the eighty controls in which self-pollination was omitted. Thus incompatible male gametes irreversibly sterilised ovules leaving only a few survivors which may have escaped fertilisation.

Thirdly, differences of compatibility in reciprocal crosses between two individuals is a feature of breeding systems in which the mating type is sporophytically determined. As an explanation of reciprocal differences in Borago this presents obvious difficulties. It cannot account for partial seed set. With sporophytic control of mating type all the gametes produced by a single individual are of like mating type which would give an all-or-nothing result. No system has yet been found to have sporophytic control of mating type in one sex and gametophytic control in the other. In Borago this is one possible explanation of the reciprocal differencies. Alternatively, epistasis may occur between mating type loci. It would be difficult to distinguish between these two kinds of gene interaction experimentally.

Finally, the overall results of crossing conform with expectations based on the theory of polygenic control of the breeding system in $B$. officinalis. 


\section{INBREEDING DEPRESSION}

Continuous inbreeding in $B$. officinalis leads to a marked decline in vigour which is expressed in various ways. The germination of seed falls off rapidly. Embryo abortion in the late stages of development as opposed to the early breakdown resulting from incompatibility increases in frequency with successive generations of selfing (fig. 5). These symptoms are accompanied by isolated cases of male sterility and morphological abnormalities including dwarf and glabrous forms. The rapid onset of severe inbreeding depression is an indication that the apparently weak outbreeding system is sufficient to prevent widespread self-fertilisation in nature.

\section{Natural outcrossing}

To measure the frequency of cross-fertilisation under natural conditions a mutant form with white flowers was used. The condition is recessive and all white flowered plants breed true.

Eighteen pairs of plants were grown together in the open at various times in widely separated locations. One member of each pair was a selfcompatible white flowered plant. The other was a true breeding blue flowered plant which was cross-compatible with its partner. Seeds from the whiteflowered parent were collected and sown. One hundred and one produced white-flowered plants, the result of self-fertilisation. Three hundred and sixty-two had blue flowers, the result of cross-fertilisation. Thus even selfcompatible plants show a high frequency of natural outcrossing.

\section{Discussion}

In $B$. officinalis the breeding system is indeterminate. Cross-mating is facultative in heterozygotes but obligatory in homozygotes. Thus the polygenic regulation of mating type is ineffective as an absolute barrier to self-fertilisation, but the homozygosity it engenders is transitory and in the end self-eliminating. Similarly complete cross-incompatibility between sibs is non-existent when their parents are unrelated, but after two generations of selfing it is complete and almost universal. Even though the defences against inbreeding are imperfect, their success in maintaining the average degree of hybridity in populations at a high level is measured by the rapid onset of severe inbreeding depression characteristic of individuals unaccustomed to excessive homozygosity.

When the optimal level of outbreeding in a population changes, polygenic incompatibility can be exploited in two directions. Firstly, selection of non-specific modifiers can reduce the effectiveness of all the mating-type loci and raise the threshold of the incompatibility reaction. By sanctioning increased inbreeding this can in turn raise the adaptive fitness of the population as a whole. A temporary amelioration of the disruptive effects of segregation and recombination can be achieved without sacrificing the potential to outbreed. Secondly, therefore, when the level of outbreeding is insufficient it can be improved either by selection of non-specific modifiers increasing the efficiency of all mating-type loci, or by selection of specific modifiers increasing the effectiveness of some loci at the expense of the rest and this may be accompanied by structural change creating a supergene 
encompassing all active loci. The second and third processes operating together could transform facultative polygenic incompatibility into a system with obligate outbreeding with one or perhaps two loci determining mating behaviour. The self-incompatible specimens of $B$. officinalis described by East (loc. cit.) may have already evolved in this way.

Bateman (1952) envisaged the existence of polygenic incompatibility as an unstable and transitory stage in the evolution of fully fledged, obligate outbreeding controlled by a single gene. Yet, the instability of the polygenic system is perhaps its greatest asset. It can delay the break up of successful existing gene combinations while conditions are stable and at the same time retain the capacity to engender new combinations to meet the demands of a changing environment. No rigid, unconditional, outbreeding system can match this unique versatility. Therefore there seems to be no reason why all outbreeding species should inevitably evolve in the direction of a genetic oligarchy when polygenic incompatibility is perhaps the ideal breeding system with almost unlimited flexibility in the regulation of recombination.

\section{Summary}

1. The genetical basis of self-incompatibility in Borago officinalis is polygenic.

2. Heterozygous individuals are self-compatible but as the number of homozygous loci increases the level of self-compatibility declines. Identity at more than a critical number of loci results in complete self-incompatibility.

3. The incompatible reaction is expressed after fertilisation.

4. In some cross-pollinations reciprocal differences in compatibility occur. These may be the result of sporophytic control of mating type in the gametes of one sex only or of epistatic interactions between mating-type genes.

5. Despite the imperfections of polygenic incompatibility as an absolute barrier to selfing, the average degree of hybridity in natural populations is maintained at a high level. This is demonstrated by the rapid onset of severe inbreeding depression and the frequency of crossing under natural conditions.

6 . The versatility of polygenic incompatibility in regulating the degree of outbreeding within wide limits is contrasted with the more rigid systems with unconditional outbreeding. It is concluded that polygenic systems have certain unique advantages and they may explain the behaviour of many self-compatible but predominantly outbreeding species.

\section{REFERENGES}

bateman, A. J. 1952. Self-incompatibility systems in Angiosperms. Heredity, 6, 285-300. CROWE, L. K. 1964. The evolution of outbreeding in plants. I. The angiosperms. Herdity, $19,435-457$.

EAST, E. м. 1940. The distribution of self-sterility in the flowering plants. Proc. Amer. Phil. Soc., 82, 449-518.

LEWIS, D., AND CROWE, L. K. 1958. Unilateral incompatibility in flowering plants. Heredity, 12, 233-256.

MATHER, K. 1941. Variation and selection of polygenic characters. 7. Genet., 32, 159-193. PANDEY, K. K. 1968. Compatibility relationships in flowering plants. Role of the S-gene complex. Amer. Nat. 102, 475-489. 